\title{
Fault Feature Extraction and Enhancement of Rolling Element Bearing in Varying Speed Condition
}

\author{
A.B. Ming, ${ }^{\mathrm{a}, \mathrm{b}}$, W. Zhang ${ }^{\mathrm{a}}$, Z.Y. Qin ${ }^{\mathrm{b}}$, F.L. Chu ${ }^{\mathrm{b}, *}$ \\ ${ }^{a} X i$ 'an High-Tech Institute, Shaanxi, Xi'an, 710025, China \\ ${ }^{b}$ State Key Laboratory of Tribology, Tsinghua University, Beijing, 100084, China
}

\begin{abstract}
In engineering applications, the variability of load usually varies the shaft speed, which further degrades the efficacy of the diagnostic method based on the hypothesis of constant speed analysis. Therefore, the investigation of the diagnostic method suitable for the varying speed condition is significant for the bearing fault diagnosis. In this instance, a novel fault feature extraction and enhancement procedure was proposed by the combination of the iterative envelope analysis and a low pass filtering operation in this paper. At first, based on the analytical model of the collected vibration signal, the envelope signal was theoretically calculated and the iterative envelope analysis was improved for the varying speed condition. Then, a feature enhancement procedure was performed by applying a low pass filter on the temporal envelope obtained by the iterative envelope analysis. Finally, the temporal envelope signal was transformed to the angular domain by the computed order tracking and the fault feature was extracted on the squared envelope spectrum. Simulations and experiments were used to validate the efficacy of the theoretical analysis and proposed procedure. It is shown that the computed order tracking method is recommended to be applied on the envelope of the signal in order to avoid the energy spreading and amplitude distortion. Compared with the feature enhancement method performed by the fast kurtogram and corresponding optimal band pass filtering, the proposed method can efficiently extract the fault character in the varying speed condition with less amplitude attenuation. Furthermore, do not involve the center frequency estimation, the proposed method is more concise for engineering applications.
\end{abstract}

(c) 2014 Published by Elsevier Ltd.

Keywords: Varying speed condition, Feature enhancement, Iterative envelope analysis, Rolling element bearings

\section{Introduction}

Rolling element bearings are one of the most widely used support elements in the rotation machinery and their failure is one of the most frequent reasons for the machine breakdowns and engineering accidents. Therefore, the condition monitoring and fault diagnostics of the rolling element bearing have attracted great number of attentions in the past several years and many powerful diagnostic techniques have been developed.

\footnotetext{
${ }^{*}$ Corresponding author

Email addresses: 79607672@qq.com (A.B. Ming), zhangweihuaiyu@163.com (W. Zhang), qinzy@mail.tsinghua.edu.cn (Z.Y. Qin), chufl@mail.tsinghua.edu.cn (F.L. Chu)
} 
Especially, the vibration-based methods enjoy a particular attention due to their non-invasive nature and their high reactivity to incipient faults. Aiming to the extraction of the fault characteristic frequency, many feature extraction methods, including envelope analysis[1, 2, 3], cyclo-stationary analysis[4, 5, 6, 7] etc., are proved to be powerful in the case of constant speed condition. However, the wide range of engineering application requires the hypothesis of constant speed to be dropped owing to the variability of loads, fluctuations of speed and transients[8]. Meanwhile, the variability of loads and transients will vary the rotating speed, which is inclined to induce faults or make the fault impulses more visible[9]. Therefore, it is of great significance for the investigation of the fault diagnosis method suitable for the varying speed condition.

In 1990, Potter and Creek[10] proposed a constant angular sampling method, named as order tracking, by the usage of the electronic impulse angular encoder and solved the frequency smearing phenomenon of the spectrum caused by the fluctuation of the rotating speed. Unfortunately, the constant angular sampling methods based on the encoder were cost consuming, complicated and inclined to be wrong in the high varying speed condition. Therefore, in 1997, Fyfe and Munck[11] developed the computed order tracking (COT) technique by sampling the signal with a constant rate and performing the angular re-sampling process by the assistance of a tachometer signal. Suppose that the shaft performances a constant angular accelerate process, the COT estimated the moments needed for the equivalent angular re-sampling at first and transformed the temporal waveform to the angular domain by interpolation methods[12].

However, the COT may make the carrier frequencies of the transient responses caused by the faults at various speed expand to a more wider scope because the nature characteristic of the bearing system hardly changes, which is not benefit for the extraction of the fault characteristic. In 2012, Cocconcelli et al.[13] proposed an original method involving the COT and the simple envelope to enhance the fault feature. Later, Borghesani et al.[14] indicated that the fault feature can be enhanced if the collected vibration is first filtered by a band pass filter before the angular re-sampling process. By applying the COT method on the envelope of the filtered signal, the proposed method solved the trouble that the carrier frequency expand phenomenon caused by the direct application of the COT on the original transient signals. It is known that a band pass filter design involves the estimation of both center frequency and band width, which are important for the efficacy of the band pass filter.

As a key part of the diagnosis procedure, the signal de-modulation of the vibration signal has been investigated for several years in the bearing fault diagnosis. Many powerful methods have been developed by introducing a band pass filter around the resonance frequency of the bearing system. In 2000, Lin and Qu[15] introduced the wavelet analysis to the band pass filter design. Based on the wavelet entropy minimization method, they varied the shape factor of the morlet wavelet to obtain the optimal band pass filter design, which successfully eliminated the noise and enhanced the fault feature. Comparatively, Su[16] optimized both center frequency and band width of the morlet wavelet using the genetic algorithm and the auto-correlation analysis was further applied on the signal obtained by the previous wavelet analysis. It is validated that the 
proposed method can efficiently depress the noise of the pass band in the application of gearbox bearing diagnosis. Combining with the sparse code shrinkage method for the in-band noise depression, He et al.[17] obtained the optimal band pass filter based on the criterion of kurtosis maximization. Bozchalooi and Liang[18] introduced the smoothness index to select the optimal parameters of the complex morlet wavelet for the de-noising. Successively, the resonance frequency estimation method was further combined with the smoothness index for the band pass filter design and successfully obtained the optimal wavelet filter[19]. In 2006, optimizing the shape factor of the morlet wavelet based on the Shannon entropy minimization criterion and the scale factor of the morlet wavelet by the usage of the singular value decomposition, Qiu et al.[20] proposed a novel weak fault diagnosis method based on the wavelet filtering. Furthermore, the wavelet decomposition and wavelet filtering were both applied on the bearing fault diagnosis, it is proven that the wavelet filtering method is more suitable for the impulse signal extraction than the wavelet decomposition method owing to the better efficiency and reliability.

Different from the wavelet analysis method, in 2006, Antoni[21, 22] proposed a formalization of the spectral kurtosis by means of the Wold Cramer decomposition of conditionally non-stationary processes and introduced to the mechanical fault diagnosis. Estimated by the ratio of the fourth spectral moment and the square of the second spectral moment of the coefficient of the short time Fourier transform, the spectral kurtosis can indicate the presence of series of transients and their location in the frequency domain. Based on the spectral kurtosis, the kurtogram was further introduced for detecting and characterizing non-stationarities in a signal, which has provided an efficient way for the optimal band pass filter design. However, relying on the assertion that each type of transient is associated with an optimal (frequency/frequency resolution) dyad $(f, \Delta f)$ which maximizes its kurtosis, and hence its detection, the complete exploration of the whole plane of the original kurtogram is a formidable task hardly amenable to on-line industrial applications. In 2007, Antoni[23] proposed a fast algorithm for computing the kurtogram over a grid that finely samples the $(f, \Delta f)$ plane. Based on the wavelet package analysis, the fast kurtogram involved an 1/3-binary tree of filter-banks and achieved a finer sampling of the (frequency/frequency resolution) plane with negligible extra computing cost. In the same year, Sawalhi[24] proposed an impulse feature enhancement method by the combination of the spectral kurtosis and the minimum entropy de-convolution(MED). It is indicated that the combination of the spectral kurtosis and MED can efficiently increase the kurtosis of the signal when it is applied on the outer fault feature enhancement of an bearing which run with the speed of $11760 \mathrm{rpm}$ [25]. However, Borghesani discovered that the kurtogram, if calculated on the analytical signal, could exactly match the sum of all peaks of the squared envelope spectrum and could suggest a possible improvement in the choice of the optimal demodulation band if the bearing fault is the only source of second order cyclostationary (CS2) components. In 2011, Barszcz and Jablonski[26] found that the center frequency of the band pass filter recommended by the fast kurtogram was far from the carrier frequency of the transient signal when the signal to noise ratio (SNR) was low, which greatly depressed the efficacy of the feature 
enhancement. They declared that the center frequency was the key point of the band pass filter design. When the center frequency estimation was accurate, the feature enhancement could be carried out even the width of the pass band was set at 3 to 5 multiple of the fault characteristic frequency. To overcome the negative effect caused by the inaccurate estimation of the center frequency, the protrugram was proposed by calculating the kurtosis of the envelope spectrum amplitudes of the filtered signal and provided an novel means of the selection of the optimal frequency band for amplitude demodulation. In 2013, inspired by the sparsity measurement used in ultrasonic non-destructive testing to improve the performance of ultrasonic signal processing algorithms, Tse and Wang[27, 28] defined the spikes with large amplitudes that reflect bearing fault signatures in the envelope spectrum as the sparse representation of a bearing fault signal in a frequency domain. Combining with the wavelet package analysis, they introduced the conception of the sparsogram by calculating the sparsity of the values of the power spectra of the envelopes of wavelet packet coefficients at different wavelet packet nodes and different packet decomposition depths. However, the proposed sparsogram may not cover a whole bearing resonant frequency band owing to the usage of the fixed center frequencies and band widths of the wavelet packet filters. Therefore, they further presented an automatic selection process for finding the optimal complex morlet wavelet filter with the help of genetic algorithm that maximizes the sparsity measurement value, which provided a special way for the pass band filter design.

It has to admit that the band pass filter design involving both center frequency and band width calculation is complex and may cause catastrophic influence on the feature improvement if the center frequency estimation is not accurate. The fast increment of the paper has also illustrated the accurate estimation of both center frequency and band width are very difficult. In order to depress the influence which may be produced by the inaccurate estimation of the center frequency of the band pass filter, a novel feature enhancement procedure is proposed by the combination of the iterative envelope analysis and a low pass filter in this paper. The iterative envelope analysis can efficiently eliminate the deterministic component of the signal and demodulate the transient response to the low pass band. Therefore, a low pass filter can efficiently eliminate the additional noise.

The rest of this paper is summarized as follows. Section 2 develops an analytical model to describe the collected vibration in the varying speed condition. Then, corresponding envelope signal is calculated in section 3. Based on the theoretical analysis, the novel feature enhancement method is proposed in section 4. Simulation and experiment studies are in section 5 and 6 to validate the proposed fault feature enhancement method. Conclusions are drawn in section 7. 


\section{Analytical model for the collected vibration}

As Ref. [29] illustrates, the vibration collected in a complicated rotor system supported by the rolling element bearings is usually composed of many different components when some faults occur. In general, the vibration components caused by such as eccentricity, bending, misalignment of the rotor or gear meshing represent as deterministic (discrete frequency) components, while the others induced by the bearing faults represent as random components. In the varying speed condition, the spectra of the vibration, including rotor eccentricity, bending, misalignment and the gear meshing, are continuous, although they still locate in the low frequency band. Because they can be changed to be discrete spectral lines in the order spectrum, they are still named as deterministic component in the varying speed condition. Therefore, neglecting the added noise, the collected vibration of the rotor system can be written as

$$
v(t)=u(t)+f(t)
$$

where, $u(t)$ denotes the deterministic components and $f(t)$ denotes be the random component. For the random component including the bearing fault vibration, the impulse responses are cyclically produced although the intervals between the adjacent impulses changes with the varying speed. Therefore, it can also be named as cyclic impulse responses. For the sake of analysis, suppose that

1. The vibration signal is only collected by one transducer, or assisted with a tachometer.

2. The rotating speed varies continuously and the vibration signal can be transformed from time domain to the angular domain with the assistance of tacho impulse series.

3. In the angular domain, the amount of the deterministic components is much less than that of the cyclic impulse responses.

\subsection{Cyclic impulse responses}

When the rotating speed changes, the interval of the adjacent impulses produced by the bearing fault, varies adaptively, although the carrier frequencies of the impulse responses seems to be constant. Referring Ref. [29], the cyclic impulse responses of the varying speed condition can still be presented by the summation of the impulse responses with different time shifts. Namely,

$$
f(t)=\sum_{k=0}^{+\infty} A_{k} \exp \left(-\zeta \omega_{\mathrm{n}}\left(t-T_{k}\right)\right) \sin \left(\omega_{\mathrm{n}}\left(t-T_{k}\right)+\phi_{k}\right)
$$

where $A_{k}$ denotes the amplitude of the $k$ th impulse response, $\zeta$ denotes the relative damping coefficient, $\omega_{\mathrm{n}}$ denotes the resonant angular frequency of the bearing system, $\omega_{\mathrm{n}}=2 \pi f_{\mathrm{n}}, T_{k}$ denotes the occurrent moment of the $k$ th impulse response, and $\phi_{k}$ denotes the initial phase angle of the $k$ th impulse response. Different from the stable speed condition, the interval of the adjacent impulses varies along with the rotating speed. 
When the fault is on the outer race, the relationship between the number and the occurrence moment of the impulse responses are given by

$$
2 \pi \int_{T_{k}}^{T_{k+1}} \frac{Z f_{\mathrm{r}}(\tau)}{2}\left(1-\frac{d}{D} \cos (\alpha)\right) \mathrm{d} \tau=\frac{2 \pi}{Z}+\Delta \theta
$$

where $Z$ denotes the amount of the rolling element, $d$ denotes the diameter of the rolling element, $D$ denotes the pitch diameter, $\alpha$ denotes the contact angle and $\Delta \theta$ denotes the angle fluctuation caused by the random slippage of the rolling element. Similarly, when the fault is on the inner race, the relationship between the number and the occurrence moment of the impulse responses are given by

$$
2 \pi \int_{T_{k}}^{T_{k+1}} \frac{Z f_{\mathrm{r}}(\tau)}{2}\left(1+\frac{d}{D} \cos (\alpha)\right) \mathrm{d} \tau=\frac{2 \pi}{Z}+\Delta \theta
$$

\subsection{Deterministic components in the varying speed condition}

From Ref. [29], it can be seen that the deterministic components usually present as powerful periodical signals with lower frequencies than the carrier frequency of the cyclic impulse responses and are modeled by the Fourier series. Similarly, in the varying speed condition, the deterministic components can be presented by the summation of the components with various frequencies, namely

$$
u(t)=\sum_{l=1}^{L} A_{l}(t) \cos \left(\int_{0}^{t} \omega_{l}(\tau) \mathrm{d} \tau\right)
$$

where $L$ denotes the total amount of the deterministic component, $A_{l}(t)$ denotes the amplitude function of the $l$ th deterministic component, and $\omega_{l}(t)$ denotes the corresponding angular frequency. It is worth noting that $A_{l}(t)$ is a slow time varying function and the analytical models, Eqs. (2) and (5), are very idealised from the physical condition. However, it can allow us to insight the phenomena of the signal with simple calculation.

\section{Envelope of multi-component signal}

Being compose of the deterministic components and the cyclic impulse responses, the collected signal is one of the multi-component signal. In order to analyse the energy change caused by the bearing fault, the envelope signal of the collected signal is calculated here. At first, the analytical form of the collected signal is given by

$$
s(t)=v(t)+j \hat{v}(t)=u(t)+f(t)+j[\hat{u}(t)+\hat{f}(t)]
$$

where, $\hat{u}(t)$ and $\hat{f}(t)$ are the Hilbert transform of $u(t)$ and $f(t)$ respectively. Then, the squared envelope of the hybrid signal is given by

$$
\begin{aligned}
|s(t)|^{2} & =v(t)^{2}+\hat{v}(t)^{2} \\
& =[u(t)+f(t)]^{2}+[\hat{u}(t)+\hat{f}(t)]^{2} \\
& =u(t)^{2}+f(t)^{2}+2 u(t) f(t)+\hat{u}(t)^{2}+\hat{f}(t)^{2}+2 \hat{u}(t) \hat{f}(t)
\end{aligned}
$$


Obviously, containing the squared envelopes of $u(t), f(t)$ and their Hilbert transforms, the squared envelope of the hybrid signal also includes the cross-terms the two kinds of signal and their Hilbert transforms, namely $2 u(t) f(t)$ and $2 \hat{u}(t) \hat{f}(t)$. Only the envelope is calculated in this paper and the instantaneous frequency is not attended, the corresponding calculation can be seen in Ref. [30].

Generally, the unit impulse response function is the product of a high frequency oscillation component and a single side exponential damping function. Therefore, suppose that the unit impulse response function satisfies the Berosian theorem, and the Hilbert transforms of $f(t)$ and $u(t)$ are given by

$$
\begin{aligned}
& \hat{f}(t)=\mathscr{H}\{f(t)\}=f(t) * \frac{1}{\pi t}=-\sum_{k=0}^{+\infty} A_{k} \exp \left(-\zeta \omega_{\mathrm{n}}\left(t-T_{k}\right)\right) \cos \left(\omega_{\mathrm{n}}\left(t-T_{k}\right)+\phi_{k}\right) \\
& \hat{u}(t)=\mathscr{H}\{u(t)\}=u(t) * \frac{1}{\pi t}=\sum_{l=1}^{L} A_{l}(t) \sin \left(\int_{0}^{t} \omega_{l}(\tau) \mathrm{d} \tau\right)
\end{aligned}
$$

where $\mathscr{H}\{\cdot\}$ denotes the Hilbert transform operator and $*$ denotes the convolution operator. Combining Eqs. (2), (5), (8) and (9), Eq. (7) can be developed as

$$
\begin{aligned}
|s(t)|^{2}= & u(t)^{2}+\hat{u}(t)^{2}+f(t)^{2}+\hat{f}(t)^{2}+2 u(t) f(t)+2 \hat{u}(t) \hat{f}(t) \\
= & \sum_{l=1}^{L} A_{l}(t)^{2}+2 \sum_{l, m=1}^{L} A_{l}(t) A_{m}(t) \cos \left(\int_{0}^{t}\left(\omega_{m}(\tau)-\omega_{l}(\tau)\right) \mathrm{d} \tau\right) \\
& +\sum_{k=0}^{+\infty} A_{k}^{2} \exp \left(-2 \zeta \omega_{\mathrm{n}}\left(t-T_{k}\right)\right) \\
& +2 \sum_{r, l=0}^{+\infty} A_{r} A_{l} \exp \left(-\zeta \omega_{\mathrm{n}}\left(2 t-T_{r}-T_{l}\right)\right) \cos \left[\omega_{\mathrm{n}}\left(T_{l}-T_{r}\right)+\left(\phi_{r}-\phi_{l}\right)\right] \\
& +2 \sum_{l=1}^{L} \sum_{k=0}^{+\infty} A_{l}(t) A_{k} \exp \left(-\zeta \omega_{\mathrm{n}}\left(t-T_{k}\right)\right) \sin \left(\int_{0}^{t}\left(\omega_{\mathrm{n}}-\omega_{l}(\tau)\right) \mathrm{d} \tau-\omega_{\mathrm{n}} T_{k}+\phi_{k}\right)
\end{aligned}
$$

From Eq. (10), it can be seen that the squared envelope of the hybrid signal is consisted of the following five parts.

1. The summation of the squared envelopes of the deterministic components

$$
P 1=\sum_{l=1}^{L} A_{l}(t)^{2}
$$

Compared with the constant speed condition, the squared envelopes of the deterministic components synthesize as the trend term of the envelope signal. Naturally, the energy of the trend term is mainly distributed around the DC offset owing to the slow time-variability of the amplitude of the deterministic component.

2. The cross terms of the deterministic components

$$
P 2=2 \sum_{l, m=1}^{L} A_{l}(t) A_{m}(t) \cos \left(\int_{0}^{t}\left(\omega_{m}(\tau)-\omega_{l}(\tau)\right) \mathrm{d} \tau\right)
$$


Similar to the constant speed condition, $P 2$ is the deterministic part of the envelope of the hybrid signal and the component amount of this part is one less than that of the deterministic components in the hybrid signal. Obviously, $P 2$ is still the summation of the time-varying functions.

3. The summation of the squared envelopes of the impulse responses

$$
P 3=\sum_{k=0}^{+\infty} A_{k}^{2} \exp \left(-2 \zeta \omega_{\mathrm{n}}\left(t-T_{k}\right)\right)
$$

Similar to the constant speed condition, this part is the summation of the squared envelopes of the impulse responses and carries the main faulty information. Apparently, containing the energies and moments of the impulse occurrence, it is very important for the bearing fault diagnosis.

4. The cross terms of the cyclic impulse responses

$$
P 4=2 \sum_{r, l=0}^{+\infty} A_{r} A_{l} \exp \left(-\zeta \omega_{\mathrm{n}}\left(2 t-T_{r}-T_{l}\right)\right) \cos \left[\omega_{\mathrm{n}}\left(T_{l}-T_{r}\right)+\left(\phi_{r}-\phi_{l}\right)\right]
$$

Compared with $P 3, P 4$ includes the coefficient of $\cos \left[\omega_{\mathrm{n}}\left(T_{l}-T_{r}\right)+\left(\phi_{r}-\phi_{l}\right)\right]$. Moreover, the summation of $A_{r} A_{l}, r \in Z, l \in Z$ is near zero, since the intervals of the adjacent impulses experience random fluctuation and the cyclic impulse responses present as the random component of the collected signal. Therefore, the energy of this part is very weak and can hardly be noticed.

5. The cross terms between the deterministic components and the cyclic impulse responses

$$
P 5=2 \sum_{l=1}^{L} \sum_{k=0}^{+\infty} A_{l}(t) A_{k} \exp \left(-\zeta \omega_{\mathrm{n}}\left(t-T_{k}\right)\right) \sin \left(\int_{0}^{t}\left(\omega_{\mathrm{n}}-\omega_{l}(\tau)\right) \mathrm{d} \tau-\omega_{\mathrm{n}} T_{k}+\phi_{k}\right)
$$

It can be seen that $P 5$ presents as cyclic impulse responses, whose carrier frequency of every impulse response is the subtraction of that of the cyclic impulse and the deterministic component in the hybrid signal. Different from the constant speed condition, the carrier frequencies of these impulse responses change with the varying of the frequency of the deterministic component.

\section{A novel feature enhancement method}

Based on the theoretical analysis, it can be seen that the component amount of the deterministic component of the envelope is one less than that of the original hybrid signal when there is no DC offset, and the energy of the deterministic component is mainly transformed to be the trend term. In this instance, the deterministic components can be iteratively cancelled by eliminating the trend term. However, different from the iterative envelope analysis in the constant speed condition[29], the deterministic component cancellation will be perform by a low-pass filter, which is used to eliminate the trend terms of the iterative envelopes. When the deterministic components are cancelled, the envelope is dominated by the envelopes of the cyclic impulse responses, which carries the fault information of the bearings. 
It is worth noting that the envelope of the impulse response is concentrated at the low frequency band. For example, if the impulse response can be modeled by the product of a single side exponential damping function and a cosine or sine function, corresponding envelope will be described by the single side exponential damping function. In this case, another low-pass filter, whose cutoff frequency is larger than the carrier frequency of the envelope of the impulse response, can be used to eliminate the noise located beyond the pass band of the low pass filter and enhance the impulse features. By referring the recommendation in Ref. [26], the cutoff frequency of this low pass filter is set to be about 3 to 5 orders of the fault characteristic frequency. Therefore, a novel fault feature extraction and enhancement procedure, suitable for the varying speed condition, is proposed here based on the combination of the improved iterative envelope analysis and a low pass filter.

Suppose that the collected vibration, involving deterministic components and cyclic impulse responses, is denoted by $x(t)$, and the $k$ th iterative envelope is denoted by $\mathrm{H}^{k}[x(t)]$. The DC offset of the collected vibration is supposed to be zero. Let $k=1$ and $\mathrm{H}^{0}[x(t)]=x(t)$,

Step 1 Calculate the envelope of signal $x(t)$. For the sake of express, the envelope signal is denoted by $y(t)$.

Step 2 Eliminate the trend term of the envelope signal by a low pass filter whose cutoff frequency is lower than the minimum of the rotating frequency. Suppose that the trend term of the envelope signal is denoted by $\tilde{y}(t)$, then

$$
y(t)=y(t)-\tilde{y}(t)
$$

Step 3 Compare the similarity between the adjacent envelopes. Let $\mathrm{H}^{k}[x(t)]=y(t)$ and calculate the cross correlation coefficient of the adjacent iterative envelope by

$$
\mu_{k}=\frac{2<\mathrm{H}^{k-1}[x(t)], \mathrm{H}^{k}[x(t)]>}{<\mathrm{H}^{k-1}[x(t)], \mathrm{H}^{k-1}[x(t)]>+<\mathrm{H}^{k}[x(t)], \mathrm{H}^{k}[x(t)]>}
$$

where $<\cdot>$ denotes the inner product operator. Based on Cauchy - Schwarz inequality, the absolute value of the cross correlation coefficient $\mu_{k}$ is between $[0,1]$. The more similar the signal, the closer to unity the cross correlation coefficient.

Step 4 Obtain the envelope of the cyclic impulse series. Compare $\mu_{k}$ with $\lambda$, a prior threshold and determine whether terminate the iteration or not. If $\mu_{k} \leq \lambda$, let $x(t)=y(t)$ and $k=k+1$, turn back to step one. Verse, terminate the iteration and output the iterative envelope by

$$
z(t)=\sqrt{<\mathrm{H}^{K-1}[x(t)], \mathrm{H}^{K}[x(t)]>}
$$

where $K$ denotes the total amount of the iteration.

Step 5 Enhance the impulse feature of the envelope signal. Filter the iterative envelope by another low pass filter whose cutoff frequency is 3 to 5 orders of the fault characteristic frequency to enhance the impulse feature. 
Step 6 Transform the envelope signal to angular domain. Re-sample the filtered envelope signal with equal angle interval by the COT method and get the envelope $z(\theta)$ in the angular domain.

Step 7 Calculate the squared order spectrum and extract the fault feature. Perform Fourier transform on $z(\theta)^{2}$ and obtain the squared order spectrum $Z(\Omega)$ of the signal. For the sake of understanding, the flow chart of the proposed procedure is shown in Fig. 1.

Fig. 1.

Apparently, the improved iterative envelope analysis (Step 1 to 4) is used to eliminate the deterministic component of the hybrid signal and extract the envelope of the cyclic impulse responses by a low pass filter with a very low cutoff frequency. Then, another low pass frequency filter with a little higher cutoff frequency is used for the feature enhancement in the Step 5. Thirdly, based on the tachometer signal, the filtered envelope dominated by the cyclic impulse responses is re-sampled with equivalent angles. Finally, the fault feature extraction is carried out on the squared envelope spectrum in the order domain. Compared with the enhancement procedures performed by a band pass filter, the proposed method is more concise and more suitable for the on line signal processing, since the low pass filter does not involve the center frequency estimation.

\section{Simulations}

In this section, simulations are used to illustrate the difficulties of the fault feature extraction in the varying speed condition and to validate the efficacy of the proposed procedure. At the same time, the procedure proposed by Borghesani et al.[14] is also applied to these simulations. Their procedure is mainly summarized at the following: At first, filter the signal with a band pass filter designed on the calculation result of the fast kurtogram. Then, calculate the envelope of the filtered signal and re-sample it with equivalent angulars. Finally, calculate the spectrum of the envelope in the angular domain and obtain the fault features.

\subsection{Simple simulation for COT shortage indication}

Without loss of generality, the bearing system is modeled as a linear single degree of freedom (SDOF) system, whose resonant frequency is $4 \mathrm{kHz}$, the relative damping ratio is 0.08 , and the ball pass frequency on the outer race (BPFO) is 5.57 multiple of the rotating frequency. In this simulation, suppose that the rotating frequency is given by

$$
f_{\mathrm{r}}(t)=15+5 \sin (10 \pi t+\pi / 2)
$$

The cyclic impulse forces applied on the bearing system to produce the cyclic impulse responses are set to be $10 \mathrm{~N}$. Pre-amplified at $80 \mathrm{~dB}$, the simulation signal is sampled at the frequency of $25 \mathrm{kHz}$ and lasts $1.2 \mathrm{~s}$. Fig. 2 illustrates the rotating frequency and the outer race fault simulation signal. It can be seen that the 
intervals of the adjacent impulse responses vary with the change of the rotating frequency. The bigger the rotating frequency, the smaller the adjacent impulse intervals. It is worth noting that the amplitude of the impulse response is deliberately set to be invariable in this simulation in order to make the influence of the COT visible on the impulse feature extraction.

Fig. 2.

The short time Fourier transforms (STFTs) of the cyclic impulse responses, in both time and angular domains, are shown in Fig. 3. It can be seen that the carrier frequencies of the cyclic impulse responses are concentrated around the resonant frequency of the SDOF system, although the impulse intervals adaptively change with the rotating frequency. Comparatively, the carrier orders of the re-sampled impulse responses vary with the oscillation of the rotating frequency owing to the varying sampling frequencies of the COT procedure and the energy of the impulse responses spreads to a more wider order scope. Such phenomena are coordinated with the condition stated by Borghesani [14]. In this case, a wider band pass filter is needed for the feature enhancement, heavier noise may be contained and the enhancement efficiency of fault feature may be degraded.

Fig. 3.

Fig. 4 illustrates the envelopes obtained by a low pass filter in time and angular domain respectively. It can be seen that the filtered envelope, carried out by a low pass filter with the cutoff frequency of $2 \mathrm{kHz}$ in the time domain, holds equivalent amplitudes, while the other one, obtained by the low pass filter with the cutoff frequency of 80 order, presents some degrees of amplitude distortion. It is indicated that a simple low pass filter with a fixed cutoff order cannot deal with the cyclic impulse responses, whose carrier orders vary as the time passing by. Therefore, the fault feature enhancement operation is recommended to be carried out in the time domain and the COT method is performed on the envelope.

Fig. 4.

\subsection{Inner race fault simulation}

In this section, an cyclic impulse responses produced by an inner race fault is simulated based on the SDOF system, whose resonant frequency is $4 \mathrm{kHz}$, the relative damping ratio is 0.1 , and the ball pass frequency on the inner race (BPFI) is 7.57 multiples of the rotating frequency. In this case, the forces caused by the inner race fault is $10 f_{\mathrm{r}}(t)\left(2+\sin \left(2 \pi \int_{0}^{t} f_{\mathrm{r}}(\tau) \mathrm{d} \tau\right)\right) \mathrm{N}$, where $f_{\mathrm{r}}(t)=10+20 t \mathrm{~Hz}$ denotes the varying rotating frequency. It is worth noting that the amplitudes of forces are set to increase gradually with the increment of the shaft speed. Sampled at the frequency of $25 \mathrm{kHz}$, the signal is pre-amplified at $80 \mathrm{~dB}$ and 
lasts $0.6 \mathrm{~s}$. Moreover, $0.5 \%$ degrees of random fluctuation are introduced and $-5 \mathrm{~dB}$ degrees of white noise are added in to make the simulation more closer to the physical condition.

Fig. 5 illustrates simulation results obtained by different procedures. The pass band recommended by the fast kurtogram is $[3124,4166] \mathrm{Hz}$. The prior threshold of iterative envelope analysis is set to be 0.95 and the cutoff frequency of the low pass filter used for the trend term elimination is set to be $0.2 \mathrm{~Hz}$. The cutoff frequency of the low pass filter used to enhance the impulse character of the signal is set to be $1 \mathrm{kHz}$. The iterative envelope analysis is terminated at the third iteration when the cross correlation coefficient reaches 0.9584 . It can be seen that both methods successfully exacted the fault feature by obtaining similar temporal envelope waveforms. However, the fault feature of the proposed method is more protrudent than that obtained by Borghesani method, because the squared order spectrum of the proposed method contains more multiples of inner race fault order than that obtained by Borghesani method. Furthermore, do not involve the estimation of the center frequency, a key parameter of the band pass filter, the proposed method is more simple than Borghesani method.

Fig. 5.

\subsection{Inner race fault simulation with a deterministic component}

In this simulation, the signal is composed of a cyclic impulse response signal produced by an inner race fault and a deterministic component used to simulate the vibration produced by the rotor unbalance. The resonant frequency of the system is $4 \mathrm{kHz}$, the relative damping ratio is 0.1 , and the BPFI is 7.57 multiples of the rotating frequency. In this case, the force caused by the inner race fault is $20 f_{\mathrm{r}}(t)\left(2+\sin \left(2 \pi \int_{0}^{t} f_{\mathrm{r}}(\tau) \mathrm{d} \tau\right)\right) \mathrm{N}$, where $f_{\mathrm{r}}(t)=10+40 t \mathrm{~Hz}$, denotes the rotating frequency. The deterministic component is given by

$$
u(t)=30 f_{\mathrm{r}}(t) \times \sin \left(160 \pi\left(10 t+40 t^{2}\right)\right)
$$

Pre-amplitude at $80 \mathrm{~dB}$, the simulated signal is sampled at the frequency of $40 \mathrm{kHz}$ and lasts $0.8 \mathrm{~s}$. Fig. 6 illustrates the simulation and results obtained by different methods. The pass band recommended by the fast kurtogram is $[6250,7500] \mathrm{Hz}$. The prior threshold of iterative envelope analysis is set to be 0.9 and the cutoff frequency of the low pass filter used for the trend term elimination is set to be $0.2 \mathrm{~Hz}$. The cutoff frequency of the low pass filter used to enhance the impulse character of the signal is set to be $1 \mathrm{kHz}$. The iterative envelope analysis is terminated at the third iteration when the cross correlation coefficient reaches 0.9140 . It can be seen that the amplitude of the simulation increases with the increment of the shaft rotating frequency and the power of the deterministic component is continuously concentrated in the band of $[900,6000] \mathrm{Hz}$. No fault feature can be observed around the resonant frequency of the bearing system. Since the fast kurtogram makes a mis-judgement of the carrier frequency in this simulation, the envelope obtained by Borghesani method includes nothing fault information. Comparatively, the iterative envelope 
analysis can adaptively eliminate deterministic components and obtain the envelope of the cyclic impulse responses with only several iterative application of the low pass filter. Moreover, the fault feature can further be enhanced by the application of another low pass filter with a cutoff frequency of three to five multiples of the fault characteristic order. Therefore, the proposed procedure can provide a more powerful feature extraction and enhancement capability when deterministic components exist.

Fig. 6.

\section{Experimental study}

In this section, the proposed method was applied to several experimental tests involving the outer race fault, the inner race fault and the compound fault of both inner and outer race fault to validate its efficacy. The experiments were carried out by the machine fault simulator, shown in Fig. 7a, in the Machine Fault Diagnostics Laboratory at Tsinghua University. The rotor shaft with an eccentric disk in the middle of the shaft is supported by two MB bearings and is driven by an AC motor through a coupling. Two loads of $5 \mathrm{~kg}$ are used to load the bearing and the whole test-bed is supported by 8 rubbers on a desk. The faulty bearing, shown as Fig. 7b and c, is located at the drive end of the rotor shaft.

Similar with the simulation, the procedure proposed by Borghesani et al.[14] is also applied to these experimental analysis to validate the efficacy of the proposed method.

Fig. 7.

\subsection{Outer race fault case}

Fig. 8 illustrates the vibration and results obtained by both methods when the shaft rotates with different speed up processes. From Figs. 8a and 8b, it can be seen that the amplitude of the impulse responses produced by the outer race fault increases with the increase of the shaft speed. The recommended frequency band of fast kurtogram used in Borghesani method is [14.336, 16.384] kHz. In the iterative envelope analysis, the threshold of cross correlation coefficient is set to be 0.90 and the cutoff frequency of the low pass filter used to eliminate the deterministic components is $0.2 \mathrm{~Hz}$. The cutoff frequency of the low pass filter used to enhance the impulse feature is set to be $2 \mathrm{kHz}$. Since the collected vibration is dominated by the outer race fault signal and the deterministic component is very weak, the iteration terminates at the second step with the cross correlation coefficient reaching 0.9241 . It can be seen that both methods successfully extract the outer race fault feature, as the first several orders of outer race fault order protrude on both order spectra. However, it is worth noting that the amplitude of the waveform obtained by the proposed procedure is in the range of $[0,40] \mathrm{ms}^{-2}$, while that of the envelope obtained by Borghesani method is in the range of 
$[0,4] \mathrm{ms}^{-2}$. Compared with the original waveform, the amplitude of the envelope obtained by the proposed procedure is more closer to the original one than that obtained by Borghesani method. Moreover, the relative protrusion degree of the discrete spectral line located at the second order of outer race fault order is relatively lower than that obtained by the proposed method.

Fig. 8.

\subsection{Inner race fault case}

Fig. 9 illustrates the vibration and results obtained by both methods when the shaft rotates with different speed up processes and the bearing experiences inner race fault. Similar with the outer race fault case, the amplitude of the impulse responses produced by the inner race fault increases with the increment of the shaft speed. The recommended frequency band of fast kurtogram used in Borghesani method is [3072, 3584] Hz. In the iterative envelope analysis, the threshold of cross correlation coefficient is set to be 0.90 and the cutoff frequency of the low pass filter used to eliminate the deterministic components is $0.2 \mathrm{~Hz}$. The cutoff frequency of the low pass filter used to enhance the impulse feature is set to be $2 \mathrm{kHz}$. For the reason that the amplitude of the deterministic component in the collected vibration signal is smaller than those of the cyclic impulse responses, the iterative envelope analysis terminated at the second iteration with a cross correlation coefficient of 0.9595 . It can be seen that both methods successfully extract the inner race fault feature, as the orders of inner race fault order companying with the order of the shaft rotating protrude on both order spectra. However, the first three orders of inner race fault order are visible on the order spectrum obtained by the proposed method, while only the first order of inner race fault order can be seen on the order spectrum obtained by Borghesani method. Moreover, it is worth noting that the amplitude of the waveform obtained by the proposed procedure is in the range of $[0,40] \mathrm{ms}^{-2}$, while that of the envelope obtained by Borghesani method is in the range of $[0,10] \mathrm{ms}^{-2}$. Compared with the original waveform, the amplitude of the envelope obtained by the proposed procedure is more closer to the original one than that obtained by Borghesani method.

\subsection{Compound fault case}

Fig. 10 illustrates the vibration and results obtained by both methods when the bearing experiences both outer and inner race faults and the shaft rotates with different speed up processes. Apparently, the amplitude of the vibration increases with the increase of the rotating speed. At the seventh second, the amplitude of the impulse response even reaches the range of $[-80,80] \mathrm{ms}^{-2}$. Compared with the condition experiencing only the outer or inner race fault, the impulse amplitudes are bigger when the bearing experienced both

Fig. 9. 
inner and outer race faults. The recommended frequency band of fast kurtogram used in Borghesani method is $[4779,5461] \mathrm{Hz}$. In the iterative envelope analysis, the threshold of cross correlation coefficient is set to be 0.90 and the cutoff frequency of the low pass filter used to eliminate the deterministic components is $0.2 \mathrm{~Hz}$. The cutoff frequency of the low pass filter used to enhance the impulse feature is set to be $2 \mathrm{kHz}$. The iteration program terminates at the second step with the cross coefficient reaching 0.9186 , because the deterministic component is very weak. It can be seen that the envelopes obtained by both methods oscillates in the range of $[0,50] \mathrm{ms}^{-2}$ and both methods successfully extract the inner and outer race fault features. Discrete spectral lines obviously protrude at the first seventh orders of the outer race fault order on the order spectrum obtained by the proposed method. Relatively, only the discrete spectral line of the first order of the inner race fault is visible. It is worth noting that the cross terms between the outer race fault order and the twice order of the rotating frequency is also visible since the twice order of the rotating frequency holds large amplitude in the order spectrum. However, only the first two orders of the outer race fault order, the inner race fault order and the rotating order protrude from the background on the order spectrum obtained by Borghesani method. It can be concluded that the proposed method is more powerful than the band pass filter performed by the fast kurtogram in the fault feature extraction and enhancement of the compound race fault analysis.

Fig. 10.

\section{Conclusions}

To drop the constant speed assumption in applications and extract the fault feature more efficiently, a novel feature extraction and enhancement procedure was proposed by the combination of the iterative envelope analysis and a low pass filtering process in this paper. Simulations and experiments were used to validate the efficacy of the proposed procedure.

Firstly, in the varying speed condition, the direct application of the COT method on the impulse response series may make the energy of the impulse responses spread to a more wider scope, which is not benefit for the feature extraction. Moreover, the feature enhancement operation performed by a low pass filter in the angular domain will induce amplitude distortion. Therefore, the feature enhancement operation is recommended to be performed in the time domain.

Secondly, replacing the DC offset subtraction operation by a low pass filtering operation, the iterative envelope analysis suitable for the constant speed condition can efficiently be extended to the varying speed condition. When the deterministic components are cancelled, the iterative envelope will be dominated by the envelopes of the impulse responses and a low pass filter can be used to enhance the impulse feature of the iterative envelope. 
Thirdly, by the application on the outer and inner race fault analysis in the experiment, the proposed procedure can efficiently enhance the fault feature with less amplitude attenuation. Furthermore, compared with the feature enhancement method performed by the fast kurtogram and corresponding optimal band pass filter, the proposed procedure do not involve the estimation of the center frequency, which is more powerful in the engineering applications.

\section{Acknowledgments}

The research work described in this paper was supported by National Science Foundation of China under Grant No. 51335006, Tsinghua University Initiative Scientific Research Program under Grant No. $2011 Z 08137$ and the State Key Laboratory of Tribology Initiative Research Program under Grant No. SKLT11A02.

\section{References}

[1] R. B. Randall, J. Antoni, Rolling element bearing diagnostics-a tutorial, Mechanical Systems and Signal Processing 25(2) (2011) 485-520.

[2] M. Feldman, Hilbert transform in vibration analysis, Mechanical Systems and Signal Processing 25(3) (2011) $735-802$.

[3] J. S. Cheng, D. Yu, Y. Yang, The application of energy operator demodulation approach based on emd in machinery fault diagnosis, Mechanical Systems and Signal Processing 21(2) (2007) 668-677.

[4] R. B. Randall, J. Antoni, S. Chobsaard, The relationship between spectral correlation and envelope analysis in the diagnostics of bearing faults and other cyclostationary machine signals, Mechanical Systems and Signal Processing 15(5) (2001) 945-962.

[5] I. Antonidais, G. Glossiotis, Cyclostationary analysis of rolling element bearing vibration signals, Journal of Sound and Vibration 248(5) (2001) 829-845.

[6] J. Antoni, Cyclostationarity by examples, Mechanical Systems and Signal Processing 23(4) (2009) 987-1036.

[7] J. Antoni, Cyclic spectral analysis of rolling-element bearing signals: Facts and fictions, Journal of Sound and Vibration 304(3-5) (2007) 497-529.

[8] C. Stander, P. Heyns, Using vibration monitoring for local fault detection on gears operating under fluctuation load conditions, Mechanical Systems and Signal Processing 16(6) (2002) 1005-1024.

[9] E. Lopatinskaia, J. Zhu, J. Mathew, Monitoring varying speed machinery vibrations-1. the use of non-stationary recursive filters, Mechanical Systems and Signal Processing 9(6) (1995) 635-645.

[10] R. W. Potter, M. Creek, Wash, Tracking and resampling method and apparatus for monitoring the performance of rotating machines (Mar. 27, 1990).

[11] K. R. Fyfe, E. D. S. Munck, Analysis of computed order tracking, Mechanical Systems and Signal Processing 11(2) (1997) $187-205$.

[12] K. M. Bossley, R. J. Mckendrick, C. J. Harris, et al, Hybrid computed order tracking, Mechanical Systems and Signal Processing 13(4) (1999) 627-641.

[13] M. Cocconcelli, L. Bassi, C. Secchi, C. Fantuzzi, R. Rubini, An algorithm to diagnose ball bearing faults in servomotors running arbitrary motion profiles, Mechanical Systems and Signal Processing 27 (0) (2012) 667 - 682. doi:http://dx.doi.org/10.1016/j.ymssp.2011.09.003.

URL http://www.sciencedirect.com/science/article/pii/S0888327011003712 
[14] P. Borghesani, R. Ricci, S. Chatterton, P. Pennacchi, A new procedure for using envelpe analysis for rolling element bearing diagnostics in variable operating conditions, Mechanical Systems and Signal Processing 38(1) (2013) 23-35.

[15] J. Lin, L. Qu, Feature extraction based on morlet wavelet and its application for mechanical fault diagnosis, Journal of Sound and Vibration 234 (1) (2000) $135-148$.

[16] W. Su, F. Wang, H. Zhu, Z. Zhang, Z. Guo, Rolling element bearing faults diagnosis based on optimal morlet wavelet filter and autocorrelation enhancement, Mechanical Systems and Signal Processing 24 (5) (2010) 1458-1472.

[17] W. He, Z.-N. Jiang, K. Feng, Bearing fault detection based on optimal wavelet filter and sparse code shrinkage, Measurement 42 (7) (2009) 1092 - 1102. doi:http://dx.doi.org/10.1016/j.measurement.2009.04.001.

URL http://www.sciencedirect.com/science/article/pii/S0263224109000773

[18] I. S. Bozchalooi, M. Liang, A smoothness index-guided approach to wavelet parameter selection in signal de-noising and fault detection, Journal of Sound and Vibration 308 (1-2) (2007) 246 - 267.

[19] I. S. Bozchalooi, M. Liang, A joint resonance frequency estimation and in-band noise reduction method for enhancing the detectability of bearing fault signals, Mechanical Systems and Signal Processing 22 (4) (2008) 915 - 933.

[20] H. Qiu, J. Lee, J. Lin, G. Yu, Wavelet filter-based weak signature detection method and its application on rolling element bearing prognostics, Journal of Sound and Vibration 289 (4-5) (2006) 1066 - 1090.

[21] J. Antoni, The spectral kurtosis: a useful tool for characterising non-stationary signals, Mechanical Systems and Signal Processing 20(2) (2006) 282-307.

[22] J. Antoni, R. B. Randall, The spectral kurtosis: application to the vibratory surveillance and diagnostics of rotating machines, Mechanical Systems and Signal Processing 20(2) (2006) 308-331.

[23] J. Antoni, Fast computation of the kurtogram for the detection of transient faults, Mechanical Systems and Signal Processing 21(1) (2007) 108-124.

[24] N. Sawalhi, R. B. Randall, H. Endo, The enhancement of fault detection and diagnosis in rolling element bearings using minimum entropy deconvolution combined with spectral kurtosis, Mechanical Systems and Signal Processing 21(6) (2007) 2616-2633.

[25] D. Wang, P. W. Tse, K. L. Tsui, An enhanced kurtogram method for fault diagnosis of rolling element bearings, Mechanical Systems and Signal Processing 35(1-2) (2013) 176-199.

[26] T. Barszcz, A. Jabłoński, A novel method for the optimal band selection for vibration signal demodulation and comparison with the kurtogram, Mechanical Systems and Signal Processing 25(1) (2011) 431-451.

[27] P. W. Tse, D. Wang, The design of a new sparsogram for fast bearing fault diagnosis: Part 1 of the two related manuscripts that have a joint title as two automatic vibration-based fault diagnostic methods using the novel sparsity measurement-part1 and 2 , Mechanical Systems and Signal Processing 40(2) (2013) 499-519.

[28] P. W. Tse, D. Wang, The automatic selection of an optimal wavelet filter and its enhancement by the new sparsogram for bearing fault detection: Part 2 of the two related manuscripts that have a joint title as two automatic vibration-based fault diagnostic methods using the novel sparsity measurement-part1 and 2, Mechanical Systems and Signal Processing 40(2) (2013) 520-544.

[29] A. Ming, W. Zhang, Z. Qin, F. Chu, Envelope calculation of the multi-component signal and its application to the deterministic component cancellation in bearing fault diagnosis, Mechanical Systems and Signal Processing 50-51 (0) (2015) 70-100.

[30] M. Feldman, Hilbert transform Applications in Mechanical Vibration, John Wiley and Sons, New York, 2011. 


\section{Figures}

Fig. 1 Flow chart of the proposed procedure

Fig. 2 Simple simulation for COT shortage indication (a) rotating frequency; (b) simulated vibration signal

Fig. 3 STFTs of the simple simulation in both time and angular domain (a) STFT of the temporal signal; (b) STFT of the angular signal; Both window length and fast Fourier transform (FFT) length is 128 point and the overlap length is 120 point.

Fig. 4 Envelopes of the simple simulation (a) envelope obtained in the time domain; (b) envelope obtained in the angular domain

Fig. 5 Envelopes of the inner race fault signal (a) original signal; (b) spectrum; (c) envelope obtained by Borghesani method; (d) squared order spectrum of (c); (e) envelope obtained by the proposed method; (f) squared order spectrum of (e).

Fig. 6 Inner race fault simulation with an deterministic component (a) original signal; (b) spectrum; (c) envelope obtained by Borghesani method; (d) squared order spectrum of (c); (e) envelope obtained by the proposed method; (f) squared order spectrum of (e).

Fig. 7 Experimental apparatus (a) machine fault simulator; (b) outer race fault; (c) inner race fault.

Fig. 8 Outer race fault case study results (a) shaft frequency; (b) original vibration; (c) angular envelope obtained by Borghesani method; (d) squared order spectrum obtained by Borghesani method; (e) angular envelope obtained by the proposed method; (f) squared order spectrum obtained by the proposed method.

Fig. 9 Inner race fault case study results (a) shaft frequency; (b) original vibration; (c) angular envelope obtained by Borghesani method; (d) squared order spectrum obtained by Borghesani method; (e) angular envelope obtained by the proposed method; (f) squared order spectrum obtained by the proposed method.

Fig. 10 Compound fault case study results (a) shaft frequency; (b) original vibration; (c) angular envelope obtained by Borghesani method; (d) squared order spectrum obtained by Borghesani method; (e) angular envelope obtained by the proposed method; (f) squared order spectrum obtained by the proposed method. 


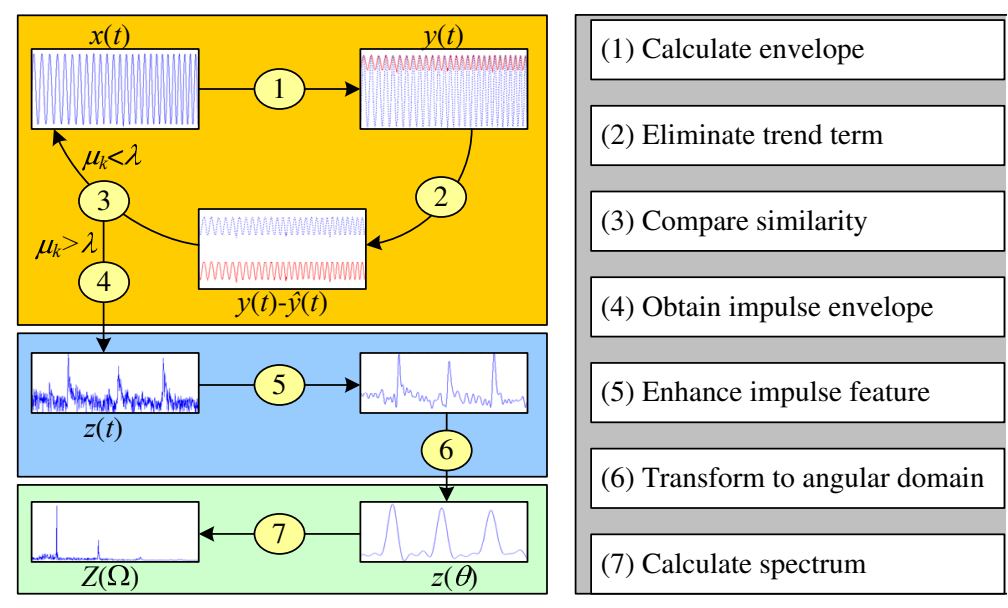

Fig. 1 Flow chart of the proposed procedure 

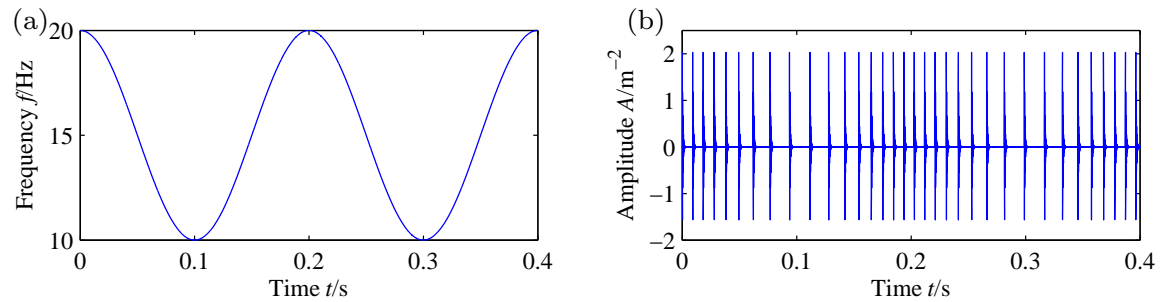

Fig. 2 Simple simulation for COT shortage indication (a) rotating frequency; (b) simulated vibration signal 

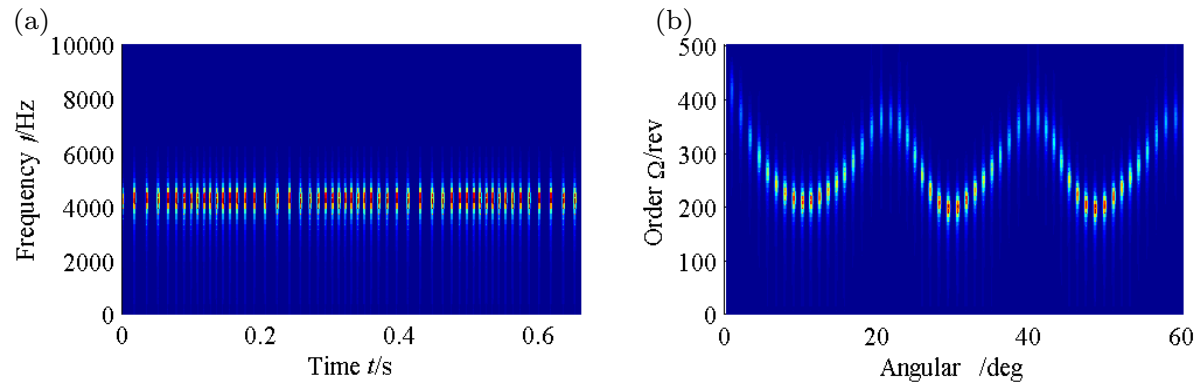

Fig. 3 STFTs of the simple simulation in both time and angular domain (a) STFT of the temporal signal; (b) STFT of the angular signal; Both window length and fast Fourier transform (FFT) length is 128 point and the overlap length is 120 point. 

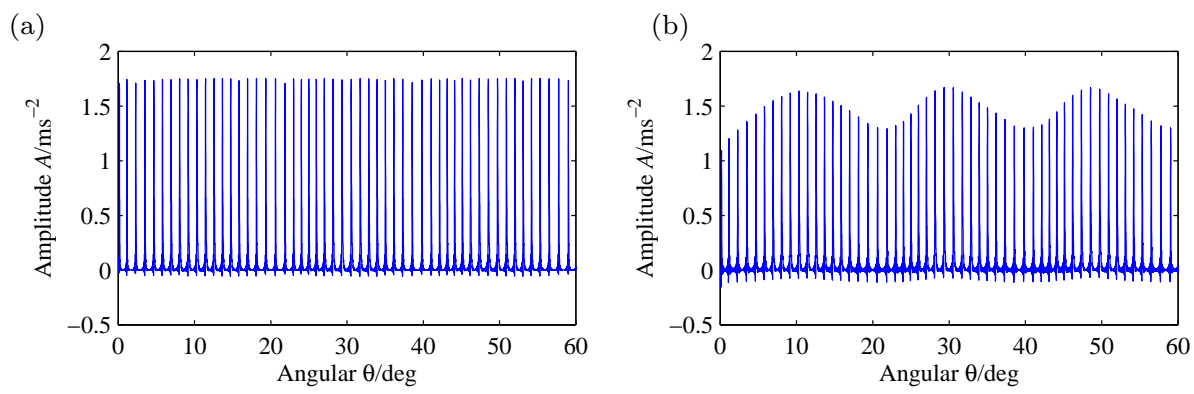

Fig. 4 Envelopes of the simple simulation (a) envelope obtained in the time domain; (b) envelope obtained in the angular domain 
(a)

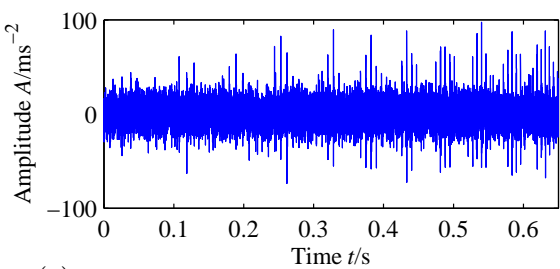

(c)
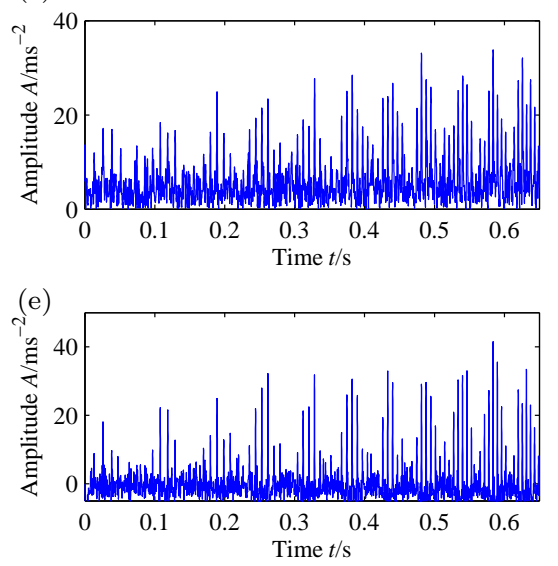

(b)

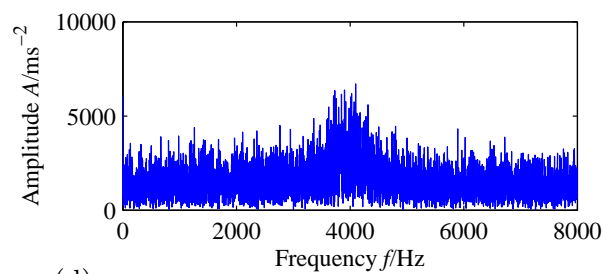

(d) $\times 10^{4}$
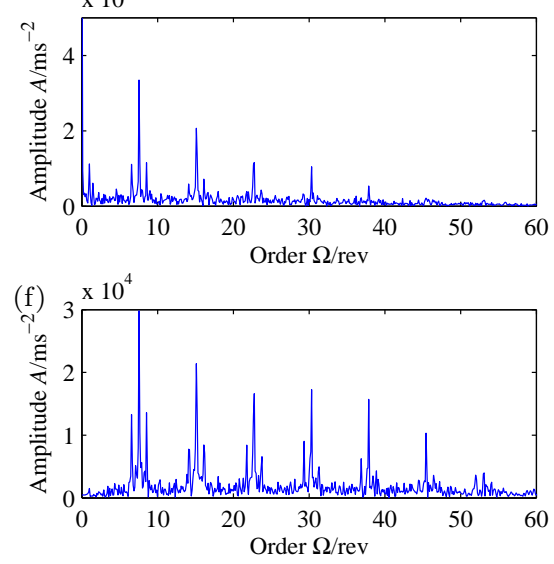

Fig. 5 Envelopes of the inner race fault signal (a) original signal; (b) spectrum; (c) envelope obtained by Borghesani method; (d) squared order spectrum of (c); (e) envelope obtained by the proposed method; (f) squared order spectrum of (e). 
(a)

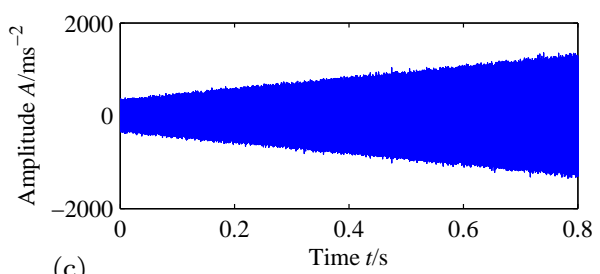

(c)
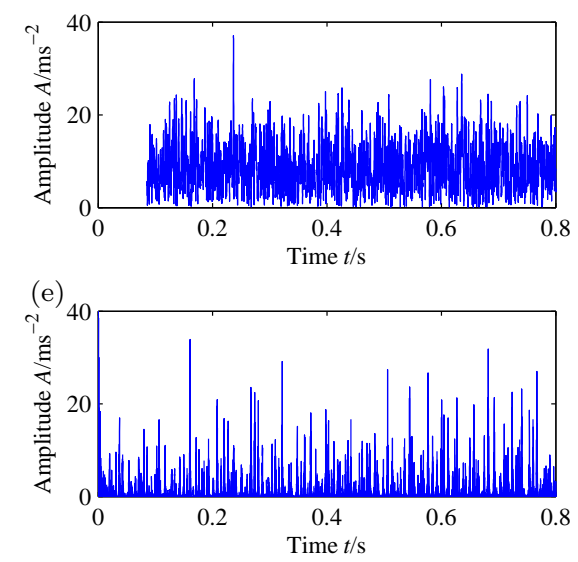

(b)

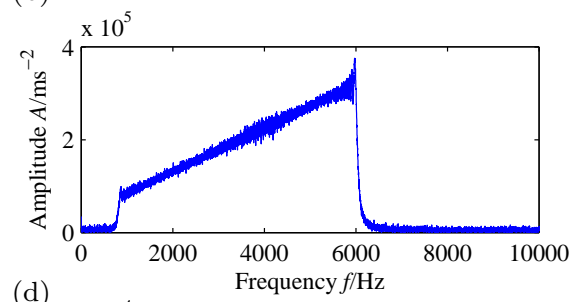

(d)
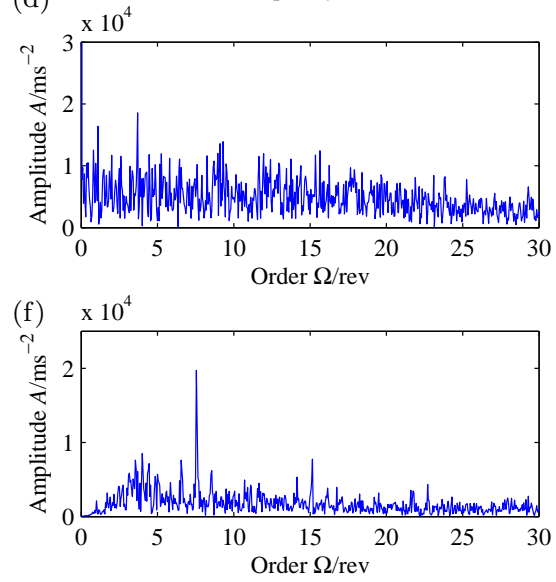

Fig. 6 Inner race fault simulation with an deterministic component (a) original signal; (b) spectrum; (c) envelope obtained by Borghesani method; (d) squared order spectrum of (c); (e) envelope obtained by the proposed method; (f) squared order spectrum of (e). 


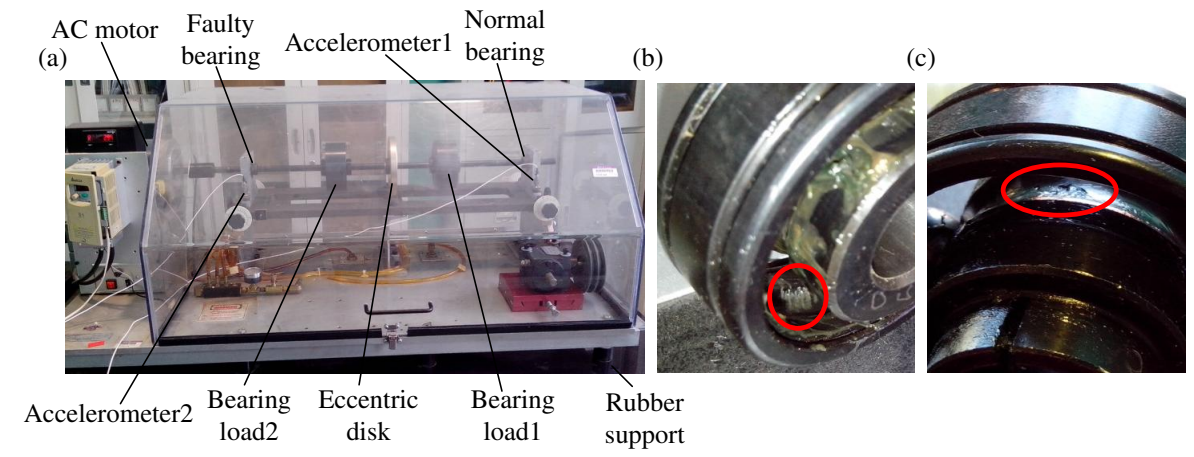

Fig. 7 Experimental apparatus (a) machine fault simulator; (b) outer race fault; (c) inner race fault. 
(a)

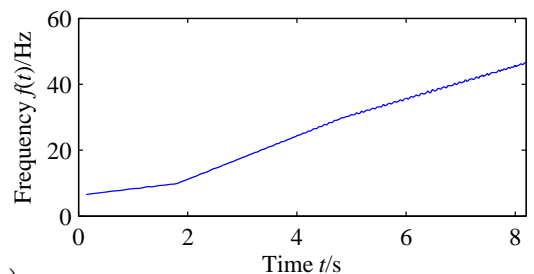

(c)

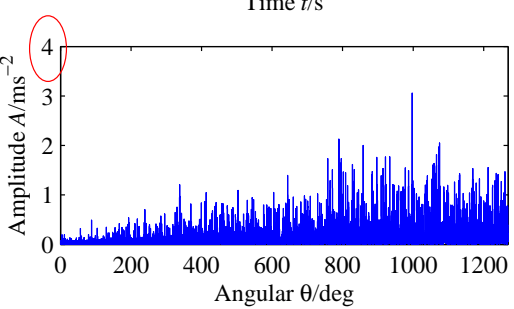

(e)

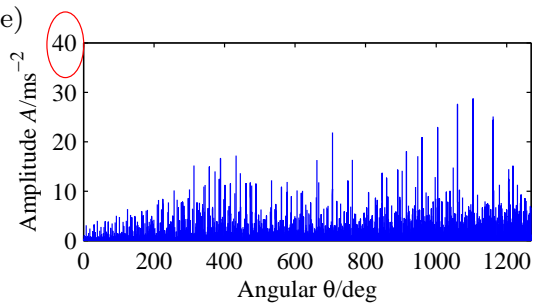

(b)

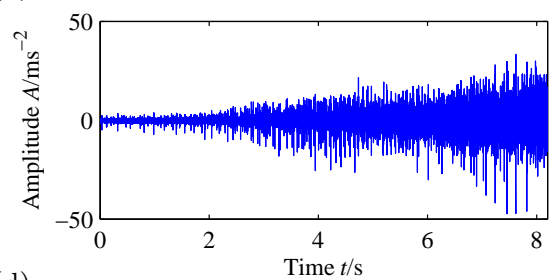

(d)

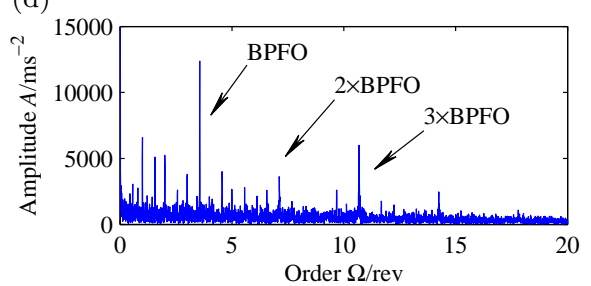

(f)

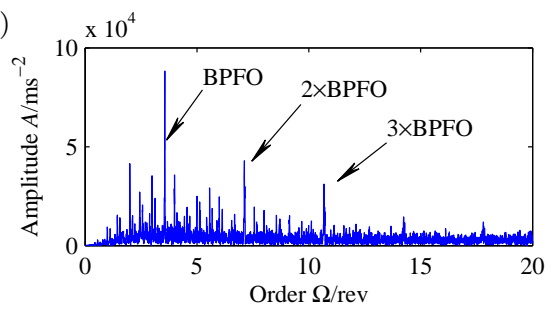

Fig. 8 Outer race fault case study results (a) shaft frequency; (b) original vibration; (c) angular envelope obtained by Borghesani method; (d) squared order spectrum obtained by Borghesani method; (e) angular envelope obtained by the proposed method; (f) squared order spectrum obtained by the proposed method. 

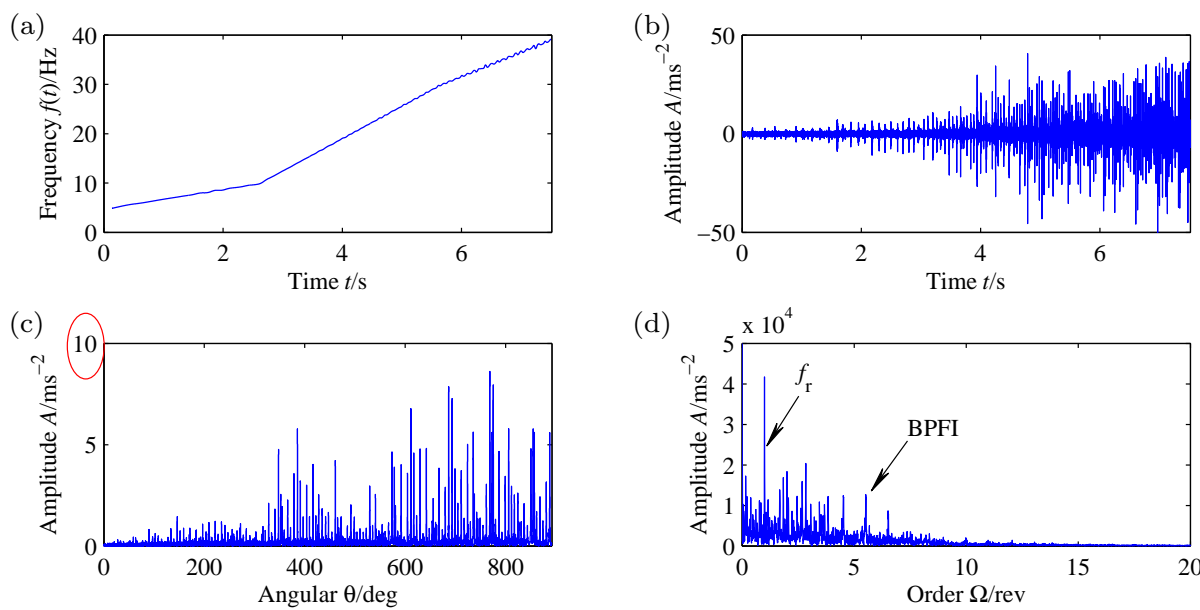

(d)
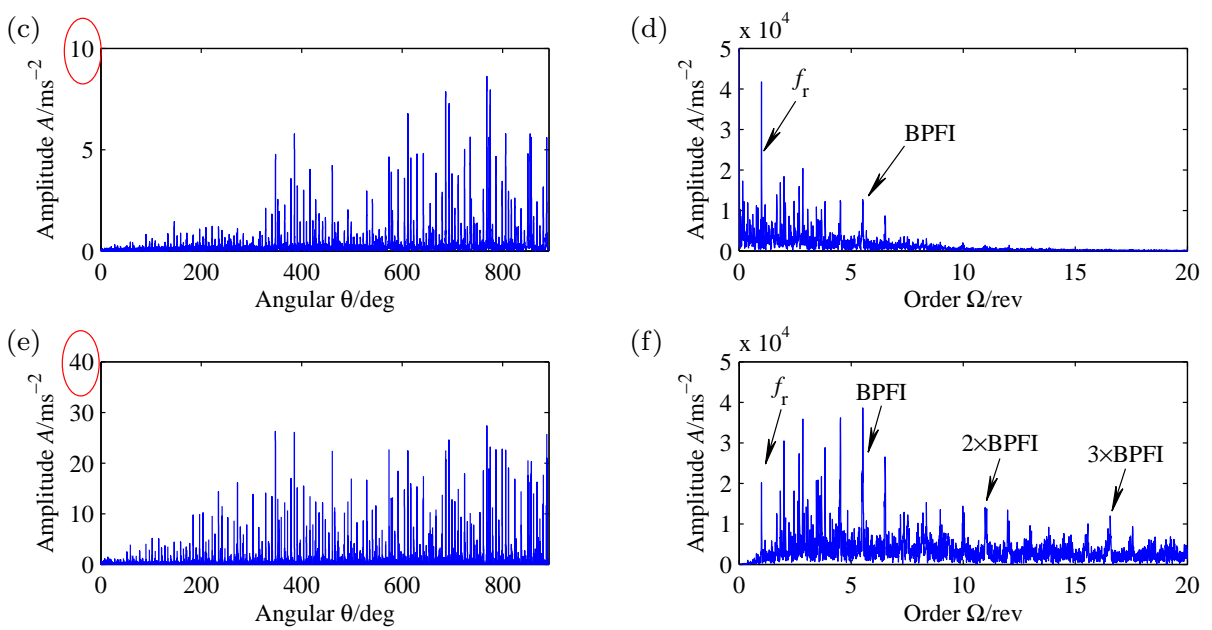

Fig. 9 Inner race fault case study results (a) shaft frequency; (b) original vibration; (c) angular envelope obtained by Borghesani method; (d) squared order spectrum obtained by Borghesani method; (e) angular envelope obtained by the proposed method; (f) squared order spectrum obtained by the proposed method. 
(a)

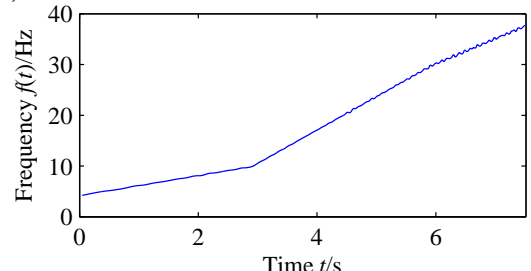

(c)

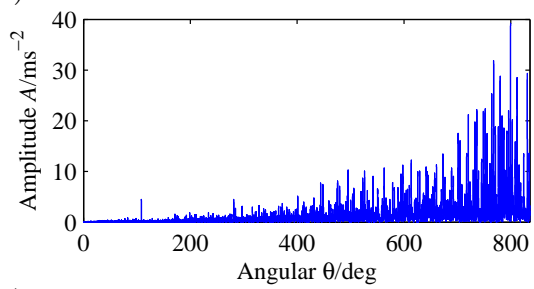

(e)

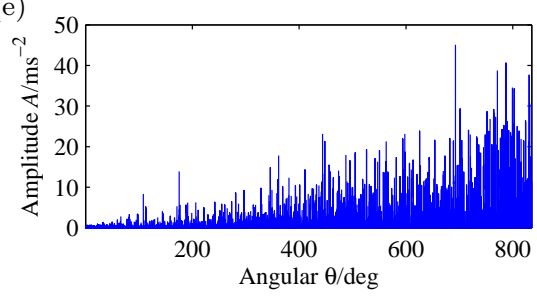

(b)

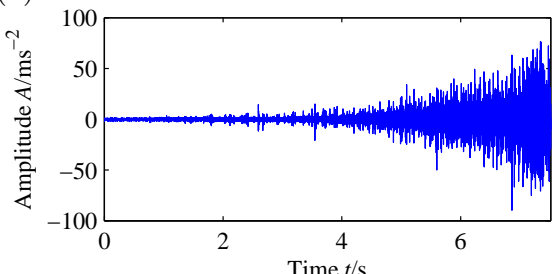

(d)

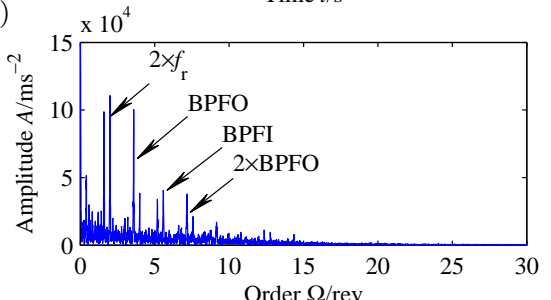

(f)

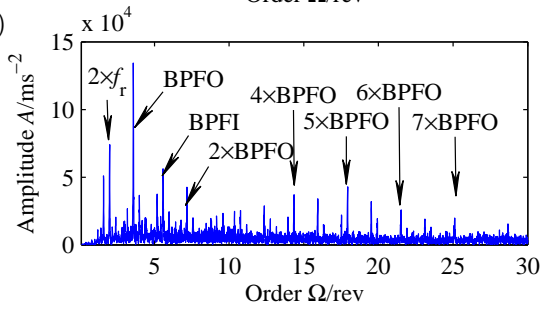

Fig. 10 Compound fault case study results (a) shaft frequency; (b) original vibration; (c) angular envelope obtained by Borghesani method; (d) squared order spectrum obtained by Borghesani method; (e) angular envelope obtained by the proposed method; (f) squared order spectrum obtained by the proposed method. 